\title{
Some Observations from the Scrutiny of Different Methods used for Iris Recognition
}

\author{
Shreyas Pathak ${ }^{1}$, Prof. Sameena Zafar ${ }^{2}$ \\ ${ }^{1}$ (Department of Electronics \& Communication, PIES/ RGPV University, India) \\ ${ }_{2}^{2}$ (Department of Electronics \& Communication, PCST/RGPV University, India)
}

\begin{abstract}
The objective of this work is to determine the ability of methods that are used for iris recognition. Because of unique characteristics of iris recognition have mesmerize more attention of researchers, which make iris identification system development rapidly. In whole process of iris identification various methods are available. Since the techniques which we are considering is the first step of iris image processing, the accuracy and quality of location will directly affect the ultimate results of iris recognition. This paper will summarize the ideas of the existed iris techniques; finally, there is a discussion on some of the challenges encountered by better techniques when operating in a real-world environment and some of the methods used to address these challenges.
\end{abstract}

Keywords: Biometrics, Iris Recognition, Haar Transform, PCA, LDA, LPCC, Gabor filter bank.

\section{INTRODUCTION}

Among biometric recognition technologies, iris recognition has attracted lots of attention because of its characteristics like uniqueness, and long term stability [1]. And iris design remains as it is from 6 months of age to the death [2]. Many works of iris recognition have been presented [3][4][5][6]. The usage of iris texture analysis for biometric identification is clearly well established. The UAE has been using iris biometrics for border control for the past decade [7] and famously claims "Some 2.7 billion Iris cross-comparisons being done every day". Even, India is using Iris as a part of its Unique ID program named "Aadhar Card Yojana", which recently passed 60 lakhs peoples enrolled. Airports in London, Amsterdam, and elsewhere continue to use iris biometrics.

\section{DIFFERENT METHODS TO RECOGNIZE IRIS}

The first stage of iris recognition is to isolate the actual iris region in a digital eye image. The iris region, shown in Fig. 1, can be approximated by two circles, one for the iris/sclera boundary and another, interior to the first, for the iris/pupil boundary. The eyelids and eyelashes normally occlude the upper and lower parts of the iris region. Also, specular reflections can occur within the iris region corrupting the iris pattern. A technique is required to isolate and exclude these artefacts as well as locating the circular iris region.

\section{A. Iris Recognition using Haar Transform}

The Comparison of Iris Code records includes calculation of a Hamming Distance (HD), as a measure of variation between the Iris Code record from the presented iris and each Iris Code record in the database.[14]

Daugman devised a test of statistical independence between two iris codes [8] and this has been implemented by many other authors including Masek [9] and Monro [10]. The Hamming Distance (HD) between the two irides to be compared is calculated. HD measures the number of identical bits between two binary bit patterns. A decision criterion based on the distribution of HDs of irides that are the same and the distribution of those that are different is determined. The overlap in these distributions determines the decision criterion. If the calculated HD between two images falls below the decision criterion, the irides are from the same person. If the calculated HD is higher, the irides are from different people. For comparing two iris codes, a nearest-neighbor approach is taken, where the distance between two feature vectors is measured using the product-of-sum of individual subfeature Hamming distances (HD). This can be defined as follows:

$$
=\left(\prod_{i=1}^{M} \frac{\sum_{j=1}^{N}\left(\text { subFeature } 1_{i j} \oplus \text { subFeature } 2_{i j}\right)}{N}\right)^{1 / M}
$$

Here, we consider the iris code as a rectangular block of size $\mathrm{M} * \mathrm{~N}, \mathrm{M}$ being the number of bits per sub feature and $\mathrm{N}$ the total number of sub features in a feature vector. Corresponding sub feature bits are XORed and the resultant $\mathrm{N}$-length vector is summed and normalized by dividing by $\mathrm{N}$. This is done for all $\mathrm{M}$ sub feature 
bits and the geometric mean of these $\mathrm{M}$ sums give the normalized HD lying in the range of 0 to1. For a perfect match, where every bit from Feature 1 matches with every corresponding bit of Feature 2, all $\mathrm{M}$ sums are 0 and so is the HD, while, for a total opposite, where every bit from the first Feature is reversed in the second, MN/Ns are obtained with a final HD of 1 .

\section{B. PCA}

Derived from Karhunen-Loeve's transformation. Given an s-dimensional vector representation of each face in a training set of images, Principal Component Analysis (PCA) tends to find a t-dimensional subspace whose basis vectors correspond to the maximum variance direction in the original image space. This new subspace is normally lower dimensional $(\mathrm{t}<<\mathrm{s})$. If the image elements are considered as random variables, the PCA basis vectors are defined as eigenvectors of the scatter matrix.[9]

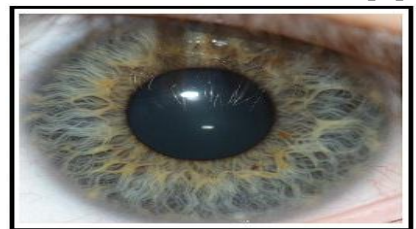

Fig. 1: Design Pattern of Iris

Below is an example of the iris dataset, which is comprised of 4 features, projected on the 2 dimensions that explain most variance:

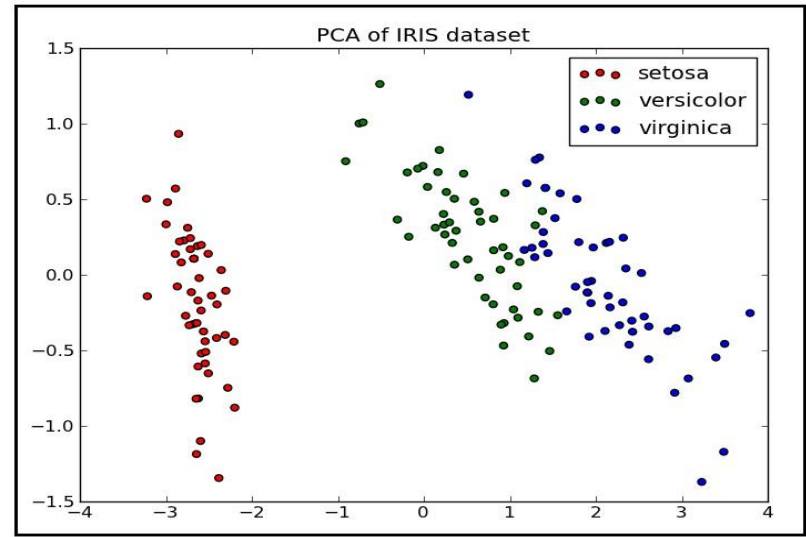

Fig. 2: Decomposing of signal using iris

\section{On Wavelet Maxima and Gabor Filter Bank}

The idea proposed uses a multiscale edge detection using wavelet maxima for iris localization followed by a Gabor filter bank decomposition for feature extraction while matching is carried out by computing the Hamming codes of different irises. The algorithms are computationally effective, reliable, robust, and the accurate. [11]

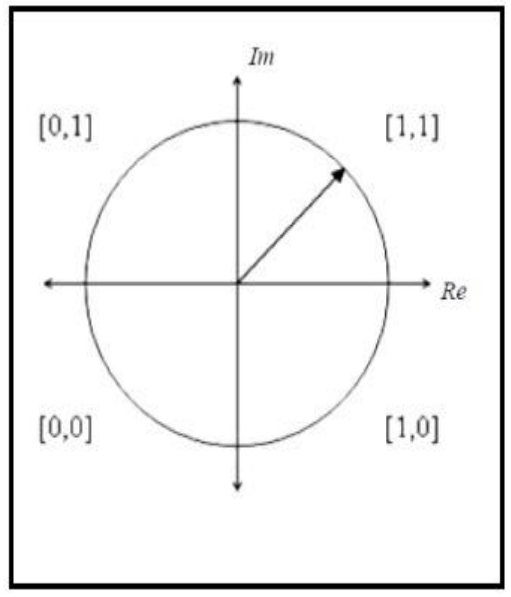

Fig.3: Phasor Quantization

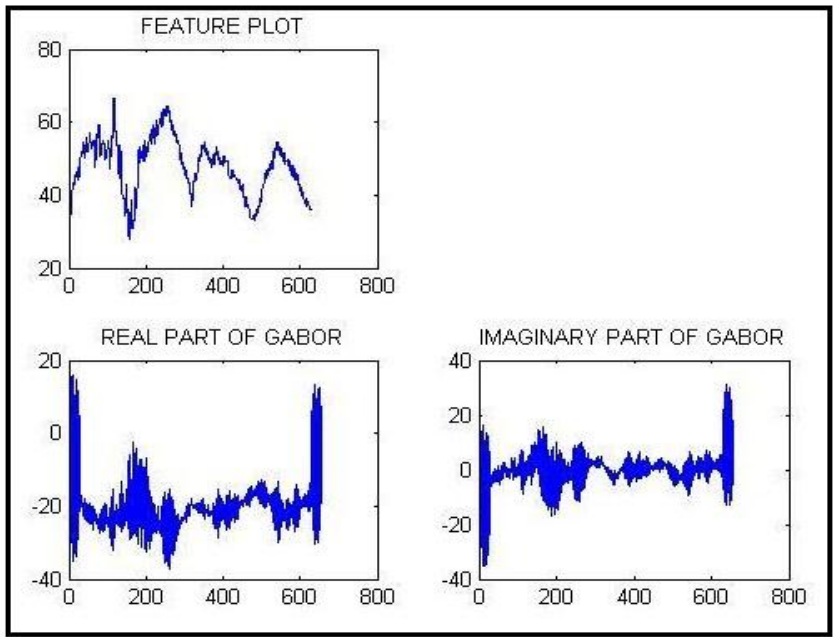

Fig.4: Gabor filter output 


\section{Phase-Based Image Matching}

Iris recognition algorithm using phase-based image matching - an image matching technique using phase components in 2D Discrete Fourier Transforms (DFTs) of given images. The use of phase components of iris images makes possible to achieve highly accurate iris recognition even for low-quality iris images. [12]

\section{High Performance Iris Recognition Based on LDA and LPCC}

In this method focus our investigation on a new iris feature extraction and representation approach to further implement an iris recognition system with low complexity and high performance.

Firstly, in order to reduce system complexity, we use 2-D wavelet transform to obtain a low resolution image and localize pupil position. By the center of pupil and the radius of pupil, we can acquire the iris circular rings. The more iris circular rings are acquired, the more information is abundant. Secondly, we adopt Sobel operator to extract iris texture. The iris texture is stretched into $1-\mathrm{D}$ array and it is regarded as a 1-D signal. The 1-D discrete wavelet transform is adopted to reduce the dimensionality of the signal. In our experiments, the wavelet permits to further reduce the system complexity. Finally, the LPCC and LDA algorithms are regard ed for feature extraction methods. A PNN (Probabilistic Neural Network) is regarded as classifier model that has proved to be effective for classification problems. The PNN is optimized by PSO. Finally, the combination of the new feature extraction methods and PNN classifier is evaluated on the CASIA iris database for iris recognition. [13]

\section{Comparisons between different methods.}

As given in the references [14] [15] [16], the algorithms were implemented in MATLAB. These algorithms have been tested on the CASIA Iris Image Database [17] As this is the only database available inpublic domain. The database includes 756 iris images from 108 individuals. For each eye, 7 images are therewhich have been captured in two sessions; three samples are collected in the first session and four in the second session. Three images for training purpose and rest of the four for testing are taken. The performance results are based on error rates: False Acceptance Rate (FAR) and False Rejection Rate (FRR); Equal Error Rate (EER) and the overall accuracy. The percentage accuracy based on FAR and FRR of the implemented algorithms is shown in Table 1.This table shows that the Daugman's algorithm [18] gives the maximum accuracy among the Eight with respect to FRR and FAR,0.01/0.09\% and overall accuracy $99.9 \%$ second highest accuracy is $99.5 \%$ given by SVM based approach proposed by Kaushik Rai.

\begin{tabular}{|c|c|c|c|}
\hline Group & EER & FAR/FRR & Overall Accuracy \\
\hline Wildes et al. & 1.76 & $2.4 / 2.9$ & 95.10 \\
\hline Avila & 3.38 & $0.03 / 2.08$ & 97.89 \\
\hline Tisse & 5.94 & $1.84 / 8.79$ & 96.61 \\
\hline Li Ma & 4.73 & $0.02 / 1.98$ & 98.00 \\
\hline Daugman & 0.95 & $0.01 / 0.09$ & 99.9 \\
\hline Boles & 8.13 & $0.02 / 1.98$ & 94.33 \\
\hline Hamed Ranjzad & 2.1 & $1.6 / 1.2$ & 98.1 \\
\hline Kaushik Rai & 0.92 & $0.03 / 0.02$ & 99.5 \\
\hline
\end{tabular}

TABLE 1. IRIS RECOGNITION METHODOLOGIES.

\begin{tabular}{|c|c|c|c|c|}
\hline $\begin{array}{l}\text { Segmenta } \\
\text { tion }\end{array}$ & $\begin{array}{l}\text { Feature } \\
\text { extraction }\end{array}$ & Accuracy & FAR & FRR \\
\hline \multirow{2}{*}{$\begin{array}{l}\text { Dougman } \\
\text { 's IDO }\end{array}$} & $\begin{array}{c}\text { Haar } \\
\text { transform }\end{array}$ & $99.97 \%$ & 0.005 & 0.01 \\
\hline & $\begin{array}{c}\text { Gabor } \\
\text { Filter }\end{array}$ & $99.94 \%$ & 0.0065 & 0.013 \\
\hline \multirow[t]{2}{*}{$\begin{array}{l}\text { Hough } \\
\text { transform }\end{array}$} & $\begin{array}{c}\text { Haar } \\
\text { transform }\end{array}$ & $99.93 \%$ & 0.01 & 0.02 \\
\hline & $\begin{array}{c}\text { Gabor } \\
\text { Filter }\end{array}$ & $99.91 \%$ & 0.012 & 0.024 \\
\hline
\end{tabular}

TABLE 2. COMPARISION OF FAR \& FRR OF DIFFERENT METHODS. 


\section{CONCLUSION}

The experimentation of Iris recognition system is tested on two databases, Bath and MMU. Initially, the preprocessing is carried with Daugman's Integro- differential operator and Hough transform localized the iris region followed by the normalization carried out by implementing a version of Daugman's rubber sheet model which eliminates dimensional inconsistencies between iris regions and itself removes eyelid, eyelash and reflection areas. Then the features of the iris are encoded by convolving the normalized iris region with Haar wavelet and Gabor filter and phase quantizing the output in order to produce a bit-wise biometric template. The Hamming distance and Euclidian distance are chosen as a matching metric, which is a measure of how many bits disagreed between two templates. The accuracy of obtained by different algorithms is as shown in above table. Segmentation by Daugman's integro-differential operator followed by the Haar transform for feature extraction and matching by Hamming distance gives maximum accuracy of $99.97 \%$. Also, with number of experimentations, the FAR and FRR are calculated and compared for different algorithms as shown in above table. The largest application of iris recognition has been in the aviation industry. Other applications are in Information Security, Security in providing access to a network, Security in providing access to a premises, Security in online business etc.

\section{REFERENCES}

\section{Journal Papers:}

[1] M Wood, N.M. Orlans, and P.T. Higgins, Biometrics, The McGraw-hill company, Berkeley, California, 2002.

[2] Kronfeld, Gross Antomy and Embryology of the Eye, The Eye,a;0 Academic Press, London, 1962.

[3] John G. Daugman, "High Confidence Visual Recognition of Persons by a Test of Statistical Independence", IEEE Trans. On 40 L Pattern Analysis and Machine Intelligence, 15(11), pp. 1148-30 I 1161, 1993,

[4] Boles, W.W. and Boashash, B., "A Human Identification Technique Using Images of the Iris and Wavelet Transform", IEEE Trans Signal Processing, 46(4), pp. 1185-1188, 1998.

[5] Li Ma, T. Tan, "Personal Identification Based on Iris Texture Analysis", IEEE Trans. on Pattern Analysis and Machine Intelligence. Vol.25, NO.12, 2003

[6] S. Lim, K. Leei, 0. Byeon, and T. Kim, "Efficient Iris Recognition through Improvement of Feature Vector and Classifier", ETRI J. vol. 23, No. 2, pp. 61-70, 2001

[7] Mohammed, Ashfaq Ahmed, Presentation on iris recognition.

[8] Byung Jun Kang and Kang Ryoung Park, "Real Time Image restoration for Iris Recognition System", IEEE TRANSACTIONS ON SYSTEMS, MAN, AND CYBERNETICS-PART B: CYBERNETICS, VOL. 37, NO. 6, DECEMBER 2007

[9] Shashi Kumar D R1, K B Raja2, R. K Chhootaray3, Sabyasachi Pattnaik, "PCA based Iris Recognition using DWT ”, IJCTA | JULY-AUGUST 2011

[10] Md. Anwar Hussain, "Eigenspace Based Accurate Iris Recognition System”, \#Department of ECE, NERIST (Deemed University) Arunachal Pradesh, India. 2010 Annual IEEE India Conference (INDICON)

[11] Makram Nabti,Ahmed Bouridane, "An Effective Iris Recognition System Based On Wavelet Maxima and Gabor Filter Bank" Institute of Electronics, Communications and Information Technology (ECIT) School of lectronics, Electrical Engineering and Computer Science, Queen's University Belfast, Belfast BT7 1NN.

[12] Kazuyuki Miyazawa, Koichi Ito, Takafumi Aoki, Koji Kobayashi, Atsushi Katsumata " AN IRIS RECOGNITION SYSTEM USING PHASE-BASED IMAGE MATCHING”, 1-4244-0481-9/06/ C2006 IEEE

[13] Chia Te CHU and Ching-Han CHEN “ High Performance Iris Recognition Based on LDA and LPCC" Institute of Electrical Engineering,I-Shou University, Proceedings of the 17th IEEE International Conference on Tools with Artificial Intelligence (ICTAI'05)

[14] S V Sheela, P A Vijaya, -Iris Recognition Methods-SurveyInternational Journal of Computer Applications (0975-8887)Volume 3 - No.5, June 2010

[15] S. Lim, K. Lee, O. Byeon, and T. Kim, -Efficient iris recognitionthrough improvement of feature vector and classifier,ETRI J.,vol. 23, no. 2, pp. 61-70, 2001.

[16] R. P. Wildes, -Iris recognition: An emerging biometric technology, Proc. IEEE, vol. 85, no. 9, pp. 1348-1363, Sep.1997.

[17] Upasana Tiwari, Deepali Kelkar,Abhishek Tiwari , "Study of Different IRIS Recognition Methods", International Journal of Computer Technology and Electronics Engineering (IJCTEE) Volume 2, Issue 1

[18] Martin-Roche D., Sanchez-Avila C. and Sanchez-Reillo R., - Iris recognition for biometric identification using dyadic wavelet transform zero-crossing\|, IEEE Aerosp. Electron. Syst. Mag., Vol. 17, Issue 10, pp. 3-6, 2002

\section{Books:}

[1] Series: Advances in Computer Vision and Pattern Recognition Burge, Mark J.; Bowyer, Kevin (Eds.) 2013, XVI, 407 p. 215 illus., 99 illus. in color. ISBN 978-1-4471-4402-1 